\title{
Molecular cloning and characterization of two differentially expressed cellulose synthase gene isoforms in Leucaena leucocephala: A pulp yielding tree species
}

\author{
Rishi K. Vishwakarma ${ }^{1 \#}$, Sameer Srivastava ${ }^{1,2 \#}$, Somesh Singh ${ }^{1}$, Bashir M. Khan ${ }^{1 *}$ \\ ${ }^{1}$ Plant Tissue Culture Division, National Chemical Laboratory, Maharashtra, India \\ ${ }^{2}$ Faculty of Medicine, Adult Cancer Program, Lowy Cancer Research Centre, University of New South Wales, Sydney, Australia \\ Email: ${ }^{\text {bm.khan@ncl.res.in }}$
}

Received 13 October 2011; revised 17 November 2011; accepted 28 December 2011

\begin{abstract}
Leucaena leucocephala is fast growing leguminous tree species, acclimatized to variety of soil and climatic conditions. It is widely used for pulp production in India. Pulp mainly consists of cellulose, which is a simple polymer of unbranched $\beta$-1, 4-linked glucan chains. The polymerization of glucose residues into a $\beta$-1, 4-linked backbone is catalysed by the enzyme cellulose synthase $(\boldsymbol{C} e s \boldsymbol{A})$. Here, cDNAs encoding Ces $A$ genes from Leucaena were isolated and characterized. The two complete cDNAs of $3.228 \mathrm{~kb}$ and $3.222 \mathrm{~kb}$ encoding $\operatorname{Ces} A$ gene from $L$. leucocephala were designated as $L$ l-7Ces $A$ (FJ871987) and Ll-8CesA (GQ267555) respectively. In-silico studies showed that $\mathrm{Ll}-7 \mathrm{Ces} A$ has $\mathbf{9 5 . 2 \%}$ identities and $\mathrm{Ll}$ 8Ces $A$ has $95.8 \%$ identities with Acacia mangium CesA2. Phylogenetic analysis revealed significant similarity with known $\operatorname{dicot} \operatorname{Ces} A$ genes. The deduced amino acid sequence of both $\operatorname{Ces} A$ genes contained the conserved D, D, D, QxxRW motif, eight membrane spanning regions and a putative zinc binding domain, which are characteristic of glycosyltransferases. DNA blot analysis suggested, $\operatorname{Ces} A$ gene to be in multiple copies in Leucaena genome. Semi quantitative and quantitative real-time PCR expression analysis of $\mathrm{Ll}$-7Ces $\mathrm{A}$ gene showed more expression in stem than leaf and not detected in root where as $L l-8 C e s A$ gene was expressed more in stem than leaf and root. Overall $L l-8 C e s A$ was expressed in all tested tissues and could be involved in active cellulose biosynthesis.
\end{abstract}

Keywords: Cellulose Biosynthesis; Cellulose Synthase; Expression Analysis; Leucaena leucocephala; Tree Species

*Corresponding author.

\#Rishi K. Vishwakarma and Sameer Srivastava contributed equally to the research article.

\section{INTRODUCTION}

Trees are reservoirs of many economically and biotechnologically significant products. Wood is one such gift of nature, which has diverse applications for mankind. One of the most well known applications of wood is paper and paper products. World paper production was around $330 \times 10^{6}$ tons per annum in the year 2003 and is increasing on yearly basis to a great extent. In India paper and pulp industry uses Bamboo, Eucalyptus and Leucaena as a major source of pulp. Leucaena is fast growing, multipurpose, tropical tree species, acclimatized to variety of soil and climatic conditions and is extensively used in India as a source of pulp [1]. The genus is native of Central America and has a wide range of usage from forage for domestic animals to fuel wood to timber and pulpwood [2].

The major constituents of wood are cellulose, hemicelluloses, and lignin. Pulp is obtained after removal of lignin and hemicelluloses from wood. Pulp consists of cellulose, which contains apparently simple linear chains of $\beta$-1, 4-linked glucan residues, but these chains aggregate to form immensely strong microfibrils. The polymerization of glucose residues into a $\beta$-1, 4-linked backbone is catalyzed by the enzyme cellulose synthase (CesA), which utilizes UDP-glucose as the substrate [3]. Despite the importance of cellulose, only recently the studies have started to unravel details of its synthesis [4]. Genes encoding plant cellulose synthases were first identified in cellulose-enriched cotton fibres [5]. With the advent of molecular approaches coupled with genome sequence information, $10 \operatorname{Ces} A$ genes have been identified in Arabidopsis [6].

The CesA gene has also been isolated from various plant species such as maize [7], rice [8], barley [9], Eucalyptus [10] and aspen [11,12]. Based on mRNA localization, in situ localization and qRT-PCR expression studies CesAs have been classified as primary or secon- 
dary wall-related CesAs $[10,13,14]$. The primary aim of above studies was to characterize the $\operatorname{Ces} A$ isoform, responsible for cell wall development.

To obtain better quality cellulose, in terms of increased degree of polymerization and crystallinity, with special reference to wood production, it is essential to identify the Ces $A$ which is directly involved in cellulose biosynthesis. Ces $A$ genes from few tree species have been isolated and characterized [11,12]. Our knowledge about $\operatorname{Ces} A$ isoforms and its activity in cellulose biosynthesis in tree species is very limited till date. With an aim to meet the increasing demand of high quality wood for paper industry, it was crucial to identify the $\operatorname{Ces} A$ isoform involved in cellulose biosynthesis from $L$. leucocephala. Here we report two different cDNA clones for CesA gene from L. leucocephala. Expression pattern of these two genes was also studied which suggests that Ll-8CesA might be more active form of CesA than $L l-7 C e s A$, expressed in all tested tissues.

\section{MATERIALS AND METHODS}

\subsection{Plant Material}

Approximately three months old cultured Leucaena plantlets were used for the analysis. To obtain cultured Leucaena plantlet, seeds of L. leucocephala were treated according to the protocol described by Shaik et al. (2009) [15].

\subsection{RNA Isolation and cDNA Synthesis}

Total RNA was isolated from L. leucocephala tissue samples (Three months old germinated seeds) according to the TRIZOL (Sigma) method. First-strand cDNA synthesis, primed with an oligo $(\mathrm{dT})_{15}$ primer, was performed with avian myeloblastosis virus reverse transcriptase (AMV RT) according to the manufacturer's protocol (Promega Corp., Madison, USA). One microgram of total RNA was used to prepare cDNA from stem, leaf and root tissue.

\subsection{Amplification of Internal Region and RACE PCR for Ces $A$ Gene}

A PCR based approach was followed to isolate the internal regions of $\operatorname{Ces} A$ gene. Known $\operatorname{Ces} A$ sequences at GenBank database were aligned using Clustal X software and primers were designed from the conserved regions. Four primer sets were designed, CesAF1, CesAF2, CesAR1, CesAR2 (Table 1). These primers were used to amplify internal regions of CesA gene. BLAST result of these amplicons showed a significant similarity to Populus, Betula and Acacia CesA genes. CesA genes from these three species were aligned and two different degenerate primer sets CesFullF1 and CesA3kR (Table 1) were designed. The primers were designed from the start codon and near to stop codon ( 3 'end) of Ces $A$ gene. The expected size fragment was sub cloned and sequenced.

Table 1. List of primers.

\begin{tabular}{|c|c|c|}
\hline S. No. & Primers Name & Primer sequence $\left(5^{\prime} \rightarrow 3^{\prime}\right)$ \\
\hline 1 & CesAF1 & GCA CCT GTT GAC ATA TTT GTC AGT AC \\
\hline 2 & CesAF2 & GAG CAA TGA AGA GAG AAT ATG AAG A \\
\hline 3 & CesAR1 & ACT CGA ACC ATT GCA TTC ATG GC \\
\hline 4 & CesAR2 & CAT ACA CTG GGC CTT GAA TTC CAT C \\
\hline 5 & CesAFullF1 & ATGGAGTCAGAAGGGGARRCYGKGGSRAAG \\
\hline 6 & CesA3kR & CGGTTCTGGCGRCCCATSARACCT \\
\hline 7 & Race Ces F & CCAAGGCGTCCGACGAAGACGGAGA \\
\hline 8 & Race Ces NF & GGTGGGTGTTGTCGCTGGGATCTCC \\
\hline 9 & CesAFull F2 & ATGGAGTCAGAAGGGGAAGCTG \\
\hline 10 & CesAFull R & CTAGCAGTTTATTCCACACTGCTC \\
\hline 11 & QCes7 F1 (Ll-7CesA) & CTC TTC ATT TCC ATC TTC GCT ACG \\
\hline 12 & QCes7 R1 (Ll-7CesA) & CAA AGT CTC CGT CTT CGT CG \\
\hline 13 & QCes8 F1 (Ll-8CesA) & TTC ATT TCC ATC TTC GCG ACC \\
\hline 14 & QCes8 R1(Ll-8CesA) & GCA GAG TCT CCA TCT TCA TCA \\
\hline 15 & CT F(5.8S rRNA) & CTA AACGACTCTCGGCAAC \\
\hline 16 & CT R(5.8S rRNA) & TTCAAAGACTCGATGGTTCAC \\
\hline
\end{tabular}


RACE PCR was attempted to isolate the full length CesA gene. Gene racer RACE kit (Invitrogen) was used according to the manufacturer's instruction. The information obtained from the amplification of internal regions of CesA gene was used to perform the RACE PCR reaction. In brief, total RNA was treated according to manufacturer's instruction and cDNA was prepared for 3' RACE reaction. 3' RACE PCR fragment was obtained after two step PCR reaction using RaceCesF and RaceCesNF primers (Table 1). The amplified fragment was purified and cloned in pGEM-T Easy cloning vector (Promega, USA). Plasmids were isolated from 10 clones and sequenced.

Sequence analysis of 3' RACE PCR clones, allowed us to design primers for full length $\operatorname{Ces} A$ gene. The following primers were designed: CesAFullF2, CesAFullR (Table 1). PCR amplification was performed using above primers and Leucaena cDNA as a template. HiFi taq DNA polymerase (Stratagene, USA) was used for the PCR reaction. The amplified fragment was cloned in pGEM-T Easy cloning vector (Promega, USA) and sequenced.

\subsection{Phylogenetic Analysis}

Ces $A$ deduced amino acid sequences from GenBank database were used to construct a phylogenetic tree. The nucleotide sequences were conceptually translated into amino acid sequence (www.expasy.ch) and evolutionary history was inferred using the Neighbor-Joining method [16]. Ces $A$ sequence from prokaryotic system Cronobacter sakazakii CesA (CrsaCes) and Mesotaenium caldariorum (MecaCesA) were chosen as an out-group. The percentage of replicate trees in which the associated taxa clustered together in the bootstrap test (500 replicates) is shown next to the branches [17]. The evolutionary distances were computed using the Poisson correction method [18] and are in the units of the number of amino acid substitutions per site. All positions containing gaps and missing data were eliminated from the dataset (complete deletion option). Phylogenetic analyses were conducted in MEGA4 [19].

\subsection{In-Silico Analysis of Two Ces $A$ Sequences}

Ll-CesA gene sequences were characterized with the Genscan software and homology was verified by database searching at the National Center for Biotechnology Information server using BLAST algorithm (http://www. ncbi.nlm.nih.gov). The deduction of the amino acid sequences, calculation of the theoretical molecular mass and $\mathrm{pI}$, was performed with ExPASy Proteomic tools provided at http://www.expasy.ch/tools/. Global alignment of two nucleotide or amino acid sequences and percentages of identity were calculated using the EM-
BOSS Pairwise Alignment Algorithms (http://www.ebi. ac.uk/emboss/). Multiple alignments of the amino acid sequences were carried out with the Clustal W1.8 program (http://www.ebi.ac.uk/clustalw/). Conserved domains in amino acid sequence were defined by searching the Pfam protein families database (http://pfam.janelia. org/) and with RPS-BLAST (Search the conserved domain database) on NCBI server. Transmembrane regions were identified using following programme TMHMM Server v.2.0 (http://www.cbs.dtu.dk/services/TMHMM/).

\subsection{Semi Quantitative and Quantitative (Real-Time) PCR}

Total RNA was extracted individually from shoot, leaf and root of three month old cultured L. leucocephala on MS media. Total RNA $(1 \mu \mathrm{g})$ was used for making cDNA. Brilliant SYBRGreen QPCR kit (Stratagene, USA) and Stratagene Mx3000P Real-Time PCR machine were used for all reactions. The primer sequences that were designed for Ll-CesAs and 5.8S rRNA is given in Table 1. Semi quantitative PCR conditions are as follows: $5 \mathrm{~min}$ at $94^{\circ} \mathrm{C}$, followed by $30 \mathrm{sec}$ at $94^{\circ} \mathrm{C}, 30 \mathrm{sec}$ at $55^{\circ} \mathrm{C}$ and $1 \mathrm{~min}$ at $72^{\circ} \mathrm{C}(25$ cycles for $5.8 \mathrm{~S}$ rRNA and for Ll-CesAs 30, 31 and 32 cycles). Last step was for $5 \mathrm{~min}$ at $72^{\circ} \mathrm{C}$. qRT-PCR reactions were performed under the following conditions: $2 \mathrm{~min}$ at $50^{\circ} \mathrm{C}$, followed by 10 min at $94^{\circ} \mathrm{C}, 40$ cycles of $30 \mathrm{sec}$ at $94^{\circ} \mathrm{C}, 30 \mathrm{sec}$ at $55^{\circ} \mathrm{C}$, $30 \mathrm{sec}$ at $72^{\circ} \mathrm{C}$. Prior to start of qRT-PCR and semi quantitative PCR reaction, equal quantity of cDNA was normalised for each reaction. Optimal numbers of PCR cycles for visible amplification for each gene were determined in preliminary experiments. Fold of expression was calculated by $\Delta \Delta \mathrm{Ct}$ method $\left(2^{-\Delta \Delta \mathrm{Ct}}\right)[20,21]$.

\subsection{Genomic DNA Isolation, Restriction Enzyme Digestion and DNA Blotting}

Good quality genomic DNA was isolated using modified protocol as described by Lodhi et al. (1994) [22]. Four different restriction enzymes namely EcoRI, BamHI, DraI and $X h o I$ were used for identifying as the multigene family of Ces $A$ gene. Standard protocol as described by Sambrook et al. (1989) [23], was followed for the experiment. In brief, Digested DNA was electrophoretically separated on $0.8 \%$ agarose gel and transfered to a Nylon Hybond-N+ membrane (Amersham, USA). Membrane was prehybridized for $8 \mathrm{~h}$ at $62^{\circ} \mathrm{C}$ in hybridization buffer (1\% BSA; $0.5 \mathrm{M} \mathrm{Na}_{2} \mathrm{HPO}_{4} \cdot 2 \mathrm{H}_{2} \mathrm{O}$, pH-7.2; 7\% SDS and $1 \mathrm{mM}$ EDTA, pH-8.0) (Sambrook et al., 1989). Hybridization was performed at $62^{\circ} \mathrm{C}$ for $18 \mathrm{~h}$ in same buffer containing radiolabeled probe $\left(\dot{\alpha}-\mathrm{P}^{32}\right)$. Common region of $\sim 800 \mathrm{bp}$ fragment was used to prepare radiolabeled probe using Amersham's random labelling kit. The blot was washed with moderate wash buffer $(2 \mathrm{X}$ 
SSC and $0.1 \%$ SDS) before exposing to Storage Phosphor Screen (GE, Healthcare) for $4 \mathrm{~h}$. The Phosphor Screen was scanned at 200 micron resolution in Typhoon Trio+ (GE).

\section{RESULTS}

\subsection{Isolation and Cloning of Two Ces $A$ Genes from $L$. leucocephala}

Amplification of internal regions of $\operatorname{Ces} A$ gene using primer combination CesAF1-CesAR1 and CesAF1-Ce$S A$ R2 gave amplification of approximately 600 and 800 bp respectively (Table 1, Figure 1). In silico study of 800 bp clones showed $88 \%$ identity with Acacia mangium and Betuala platyphylla, and $86 \%$ with Populus trichocarpa nucleotide sequences. Above three sequences were aligned and degenerate primers were designed from start codon (CesAFullF1) and near 3' end (CesA3kR) of the $\operatorname{Ces} A$ sequence (Table 1, Figure 1). Approximately $3.0 \mathrm{~kb}$ amplicon was amplified and cloned in pGEM-T Easy vector and sequenced. Sequence analysis of the clones led us to conclude the possibility of two different $\operatorname{Ces} A$ genes. Now as 5' end was known, 3' RACE was performed to reach the 3' end of the CesA coding sequence. Sequencing of 10 3' RACE clones again suggested presence of two CesA isoform in Leucaena and allowed us to design primers (Ces $A$ Full F2 and Ces $A$ Full R) for amplification of full length $\operatorname{Ces} A$ coding region (Table 1, Figure 1). An approximately $3.2 \mathrm{~kb}$ fragment was amplified, cloned and sequenced. Two different types of cDNA sequences were designated as Ll-7CesA and Ll-8CesA (GenBank Accession No. FJ871987 and GQ267555) respectively. Identities and similarities between both $L$ l-CesAs were $96.3 \%$ and $97.6 \%$ respectively.

\subsection{Molecular Characterization of $L l-7 C e s A$ and Ll-8CesA Gene from $L$. leucocephala}

Like other plant cellulose synthase both Ll7-CesA and Ll-8CesA proteins contains the D, D, D, QxxRW (QVLRW) motifs respectively (Figure 2(a)), which is a characteristics of beta-glycosyltransferases [11,24]. The regions surrounding the $\mathrm{D}, \mathrm{D}, \mathrm{D}, \mathrm{QxxRW}$ residues shows similarity between other cellulose synthases and is known as U1, U2, U3 and U4 domains [24,25]. There are eight highly conserved cysteine residues in four pairs of $\mathrm{CxxC}$ in $\mathrm{N}$-terminal region of both $L l$-CesA proteins (CxxC motif underlined) were observed. These residues form the putative LIM like zinc-binding domain [26]. Like other cellulose synthases these two CesAs from Leucaena also have six transmembrane domains in carboxy terminal region of the protein and two transmembrane domains (T1-T8) in the amino terminal region. The amino terminal region of $\mathrm{Ll}$-Ces $\mathrm{As}$ protein also contains SPXX motifs (Figures 2(a) and (b)) that are characteristics of nucleic acid binding proteins [27].

\subsection{Ll-CesA: A Multigene Family}

To investigate the multigene nature of CesA genes in $L$. leucocephala, DNA blot analysis was performed using genomic DNA. Good quality genomic DNA was isolated and digested with following restriction enzymes: EcoRI, DraI, XhoI and BamHI. Out of these restriction enzymes Bam HI and DraI cuts inside the Ll-CesA gene. Radioactive probe of approximately $800 \mathrm{bp}$ was prepared from a common region of both $L l$-CesAs cDNA sequence. Multiple bands were seen on blot in all four lanes; which clearly suggest that existence of multiple copies of Ces $A$ gene (Figure 3). The presence of multiple copies of CesA in Leucaena was in coherence with other known plant species.

\subsection{Ll-CesA Genes: Its Similarity to Ces As Involved in Cellulose Biosynthesis}

An unrooted phylogenetic tree was drawn to investigate the evolutionary and similarity index of Ll-CesAs with homologous $\operatorname{Ces} A$ sequences by using deduced amino acid sequences. Ll-7CesA and Ll-8CesA from Leucaena share a $95.8 \%$ and $95.2 \%$ identity and $97.8 \%$ and $96.9 \%$ similarity with Acacia; $90.8 \%$ and $89.7 \%$ identity and 95.3\% and 94.2\% similarity with Populus and $89.4 \%$ and $88.4 \%$ identity and $93.3 \%$ and $92.4 \%$ similarity with Eucalyptus respectively. The tree was divided into four clusters. Cluster I subdivided into two groups. The group I comprises monocotyledonous plant species and group II comprises dicotyledonous plant species. Both L1-7CesA and Ll-8CesA were grouped with CesAs of dicotyledonous

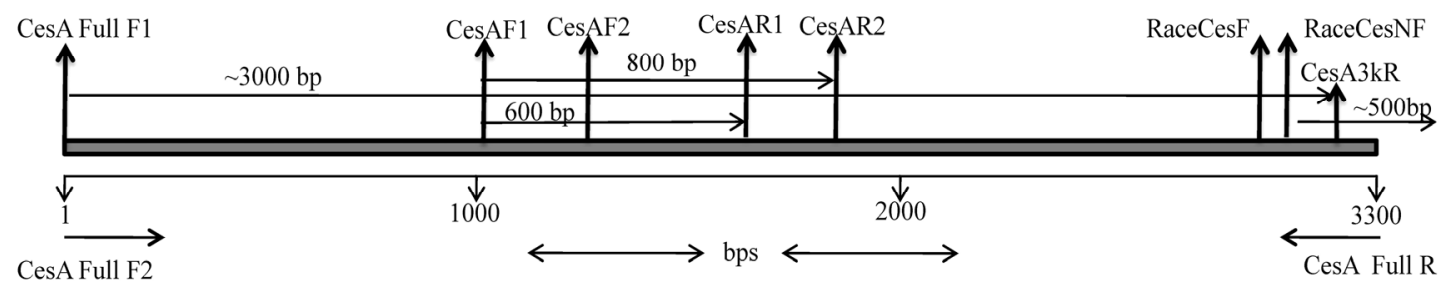

Figure 1. Schematic representation of designing of primers and expected amplicon sizes: Start sites of primers used for isolation of $L l$-Ces $A$ genes. 


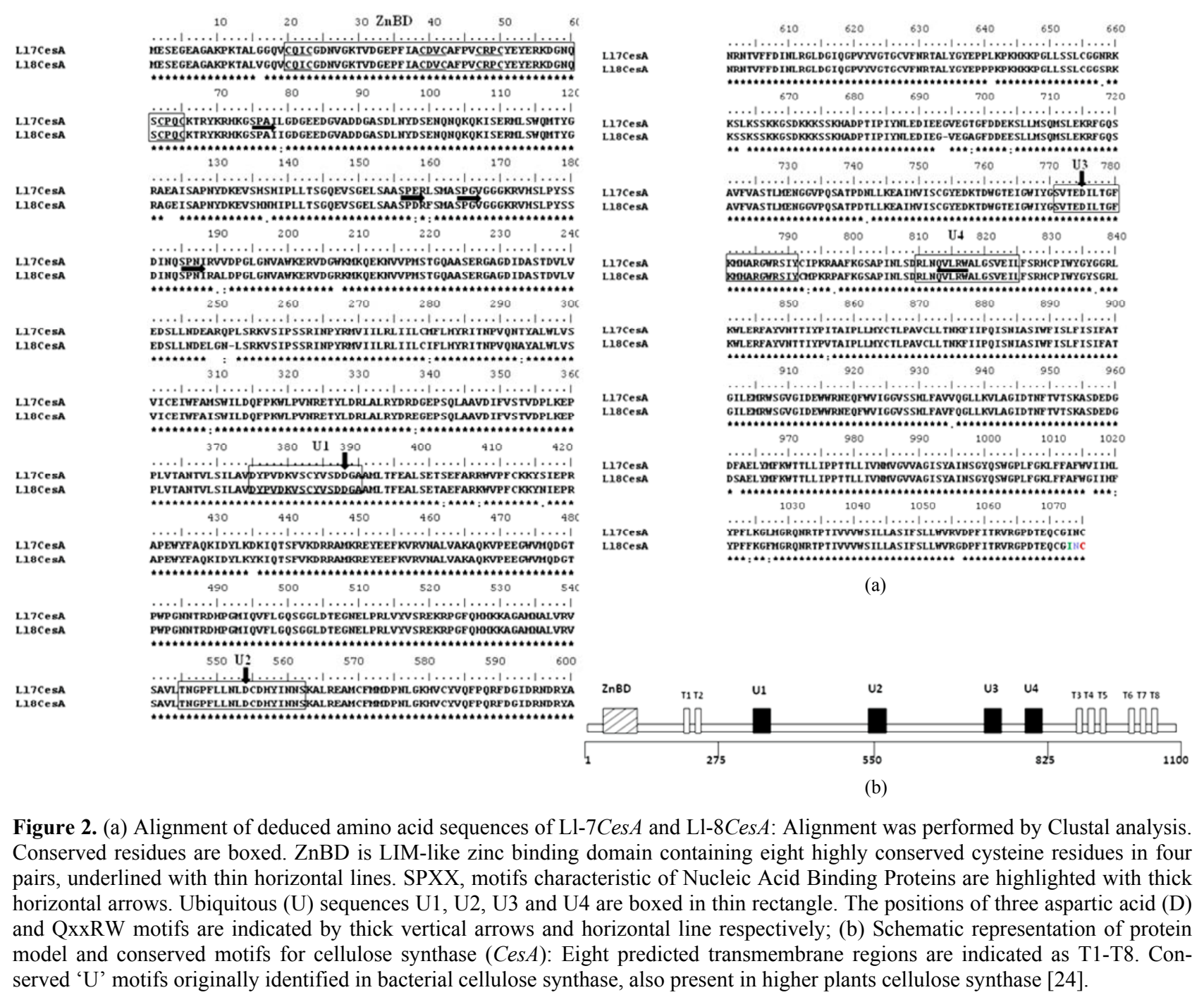

species Eucalyptus, Populus and Acacia in group II of cluster I (Figure 4).

\subsection{Tissue Specific Differential Expression of Two Ll-Ces A Genes}

The expressions of both $L l$-CesAs were determined using semi quantitative PCR and quantitative real-time PCR (qRT-PCR). The expression patterns of Ll-CesAs genes were examined in shoots leaves and roots. Semi quantitative PCR suggested that both the genes are differentially expressed. Equal quantity of cDNA was determined by 5.8S rRNA primers (Figure 5, lane A). The expression of $L l-7 C e s A$ was observed in stem and leaf only. However expression of Ll-7CesA in shoots was higher than leaves (Figure 5, lane B). In case of $\mathrm{Ll}$ 8 Ces $A$, expression level was observed highest in shoot than leaves and roots (Figure 5, lane C). Moreover the expression level of $L l-8$ CesA was comparatively higher than $L l-7 C e s A$. qRT-PCR was used for determining fold 


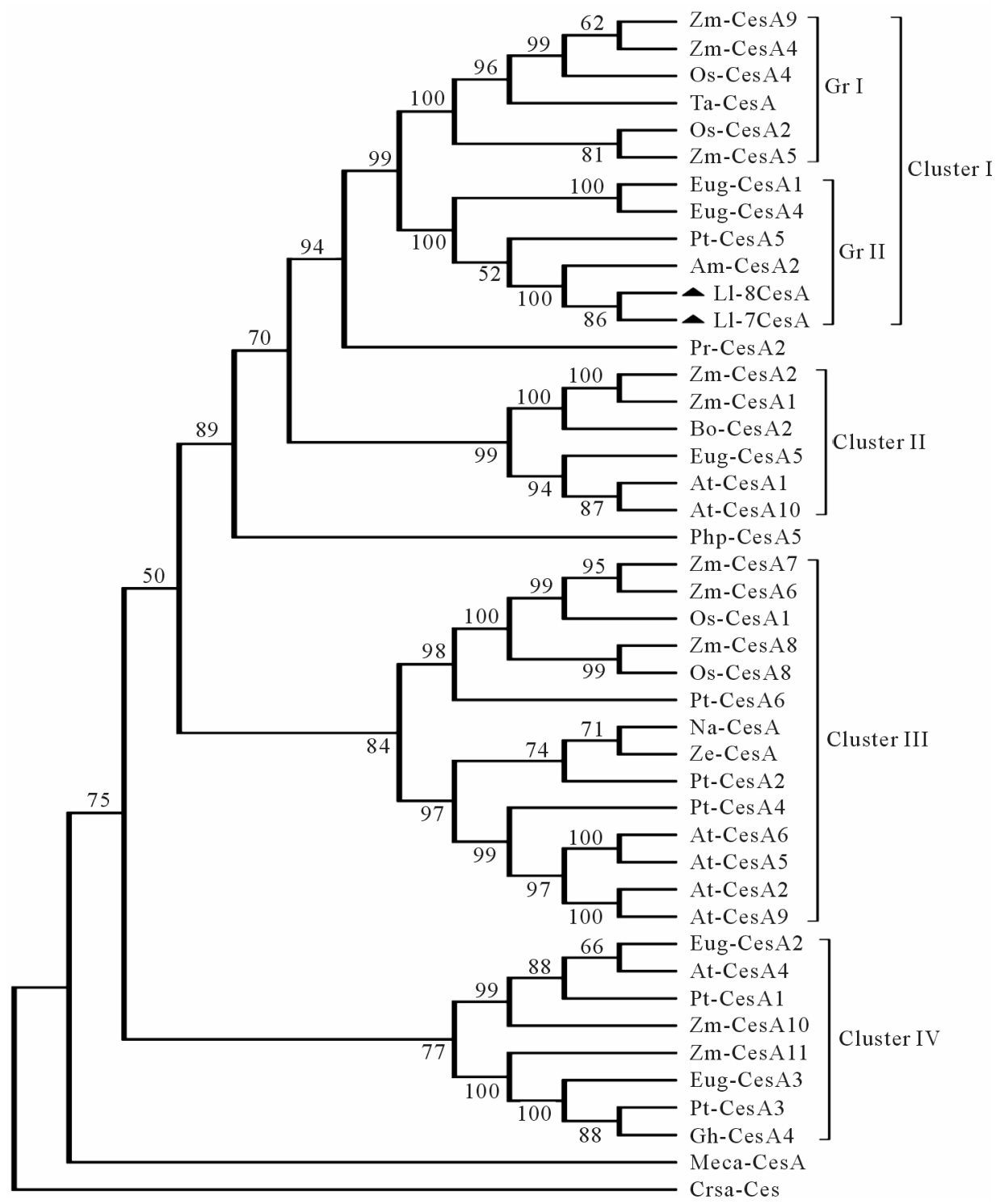

Figure 4. Evolutionary Relationship of $L l$-CesAs among various CesA proteins: Unrooted Neighbor-joining Phylogenetic tree of Ll-CesAs: The phylogenetic tree was constructed on the basis of ClutalX2 multiple sequence alignment of deduced amino acids of Ll-CesAs with 42 CesA protein sequences. For development of tree 500 bootstrap replicates and only branches with $50 \%$ or greater support were considered. Species were abbreviated as follows and accession numbers as well as type of CesAs are given in brackets. Arabidopsis thaliana (AtCesA1-NP_194967; AtCesA2-NP_195645; AtCesA4-NP_199216; AtCesA5-NP_196549; AtCesA6-NP_201279; AtCesA9-NP ${ }^{-}$179768; AtCesA10-NP 180124); Zea mays (Z̄mCesA1-NP 001104954; ZmCesA2NP 001105574; ZmCesA4-NP 001105621; ZmCesA5-NP 001104955; ZmCesA6-NP 001104956; ZmCesA7-NP_001104957; ZmCesA8-NP_001104958; Z̄mCesA9-NP_001104959; ZmCesA10NP_001105672; ZmCesA11-NP_001105236); Ōryza sativa (OsCesA1-NP_001051830; OsCesA2NP_001051648; OsCesA4-NP_001059162; OsCesA8-NP_001059303); Populus tremula (PtCesA1AAT09894; PtCesA2-AAT09895; PtCesA3-AAT09897; PtCesA4-AAT09898; PtCesA5-AAL23710; PtCesA6-AAP40636); Eucalyptus grandis (EugCesA1-ABY25277; EugCesA2-ABY25278; EugCesA3-ABY25279; EugCesA4-AAY60846; EugCesA5-AAY60847); Triticum aestivum (TaCesA-BAD06322); Mesotaenium caldariorum (MecaCesA-AAT48369); Nicotiana alata (NaCesA-AAK49454); Ze-Zinnia elegans (ZeCesA-BAG06272); Gossypium hirsutum (GhCesA4AAL37718); Leucaena leucocephala (Ll-7CesA-ACU87559; Ll-8CesA-ACU80553); Acacia mangium (AmCesA2-AAT66941); Pinus radiata (PrCesA2-AAQ63936); Bambusa oldhamii (BoCesA2-AAY43218); Physcomitrella patens (PhpCesA5-ABI78958); Cronobacter sakazakii (CrsaCes-YP_001440220). 


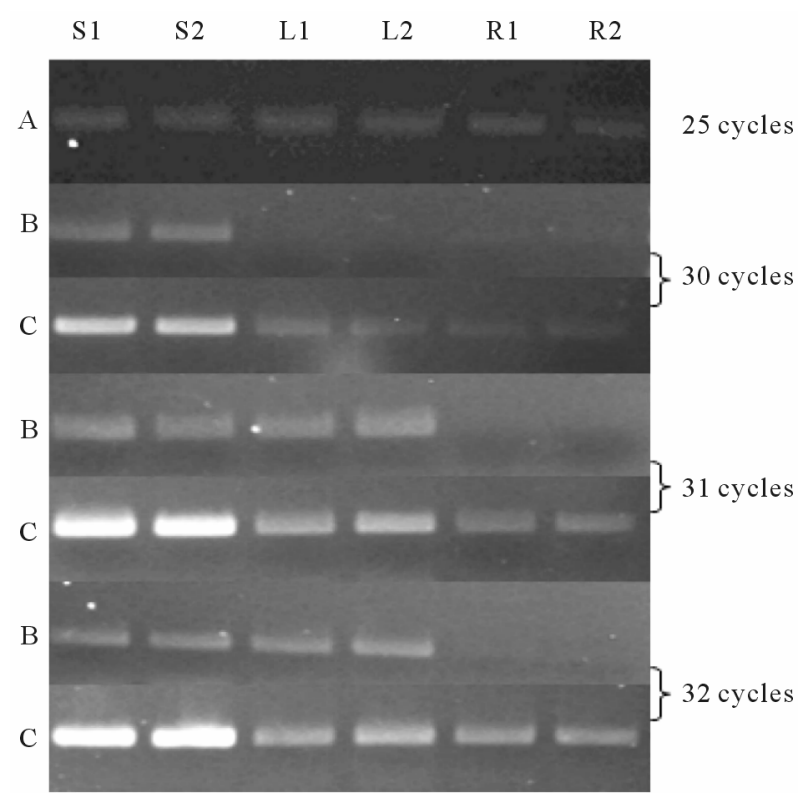

Figure 5. Semi quantitative PCR analysis: PCR was done at three different cycle numbers $(30,31$ and 32 cycles) with cDNA from shoot (In duplicate, S1 and S2), Leaf (L1 and L2) and Root (R1 and R2); observed on $2 \%$ agarose gel. Lane B and Lane C: Ll-7CesA and Ll-8CesA expression respectively. Lane A: 5.8S rRNA (25 Cycles).

expression of both the Ll-CesAs genes in different tissues. No Ct value was recorded for Ll-7CesA in case of root. When compared to leaves, shoots have 5.35 fold more expression than leaves (Figure 6(a)). However In case of $L l-8 C e s A$, least expression was recorded in roots. The expression was 15.03 fold higher in shoots and 1.51 fold more in leaves when compare to roots (Figure 6(a)). Moreover when expression of individual genes were compared in individual tissues then it was found that Ll-8CesA was 10 fold higher in case of shoot and 5.3 fold higher in case of leave tissue when compared to Ll-7CesA expression (Figure 6(b)).

\section{DISCUSSION}

It has been established in Arabidopsis, Zea mays and Oryza sativa that CesA is a multigene family $[6,8,14,28]$. Reports have also described that these genes perform individual function such as development of primary and secondary cell wall. On the contrary, presence of more than two sequences in the genome of Eucalyptus, Populus, Arabidopsis, maize and barley for CesA suggests that Ces $A$ genes might be involved in performing redundant functions inside the cell [7,26,29-31]. Compared to primary walls, secondary walls contain higher amounts of cellulose with a higher degree of polymerization and crystallinity [25,32]. Therefore, characterization of CesA gene, primarily responsible for development of secondary cell wall is of paramount importance in gener-

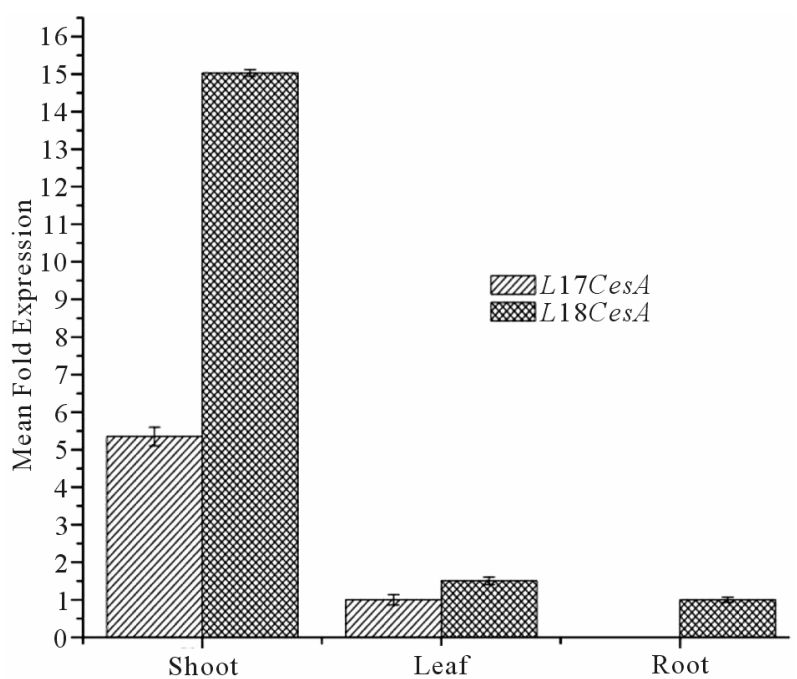

(a)

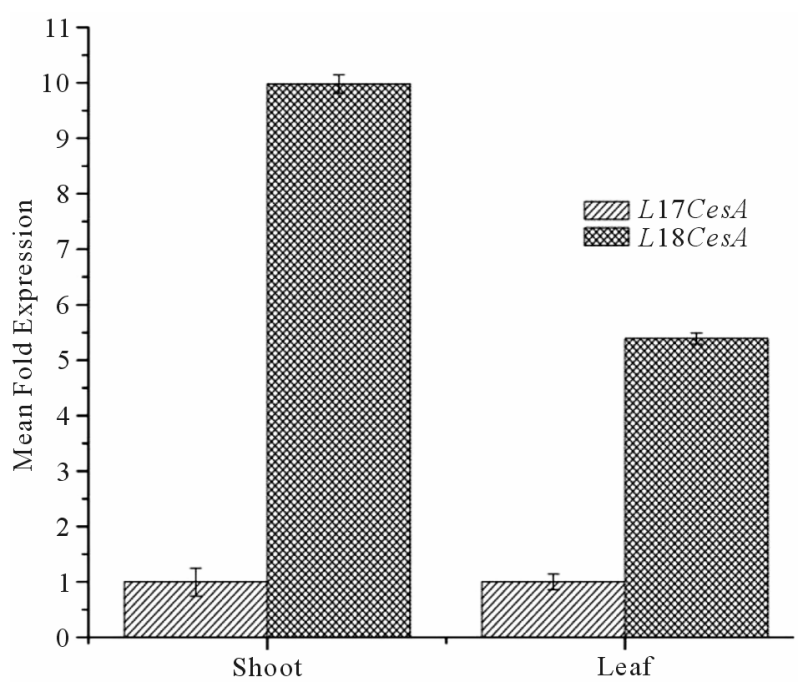

(b)

Figure 6. (a) Tissue specific normalized expression of $L l-7 C e s A$ and $L l$-8CesA using qRT-PCR: Light grey shaded histogram represents mean fold expression of $L l-7 C e s A$ and dark grey histogram represents mean fold expression of $L l-8 C e s A$; (b) Comparison of mean fold expression level of $L l-7 C e s A$ and $L l-8 C e s A$ transcripts in individual tissues: Light grey histogram: Ll-7CesA, Dark grey histogram: Ll-7CesA. Error bars are showing standard deviations (SD) for triplicate analysis.

ating elite tree species for paper industry.

With an aim to characterize CesA gene responsible for development of secondary wall, two full length CesA genes of approximately $3.2 \mathrm{~kb}$ were isolated from $L$. leucocephala. They possess $96.3 \%$ identity and $97.6 \%$ similarity with each other. Size of the cDNA was comparable to known sequence of CesA from other plant species. Primary in-silico analysis showed that both the cDNA sequences are similar to other known homologous CesA sequences. DNA binding, transmembrane, D, D, D, 
QxxRW, SPXX, CxxC motifs were identified in both the cDNA sequences. All these data classifies them to be member of family glycosyltransferase (Figure 2). Phylogenetic analysis also revealed that both the cDNAs share a significant similarity with $\operatorname{Ces} A$ sequence from other dicot tree species. Ll-7CesA and Ll-8CesA were grouped with Eucalyptus and Populus which have dual nature and are involved in primary as well as secondary cell wall biosynthesis which suggest that these sequences might be involved in such functions in L. leucocephala (Figure 4).

Multigene nature of CesAs has been well established in various research articles, Our DNA blot results also suggest that CesAs are present as a multigene family in Leucaena. Presence of multiple bands in the blot suggests that the CesA is present in multiple copies in Leucaena genome (Figure 3). Similar results have also been cited in model plants such as Arabidopsis, rice and poplar [8,33-38].

Now the question was where these transcripts are being expressed. We performed a semi quantitative PCR paralleled with qRT-PCR to investigate the presence of Ll-7CesA and Ll-8CesA transcript in various tissues. Root, stem and leaves were investigated for this experiment. The expression level of $L l-8 C e s A$ was higher than Ll-7CesA in all tissues tested. However the overall expression of individual transcript was highest in stem than leaves and roots in both the case. Differential expression pattern of CesAs in Leucaena corroborated with the expression pattern of other homologous CesA transcript. When compared to CesAs of Eucalyptus, Arabidopsis and Populus, it was found that the expression pattern of both $L l$-CesAs is similar to primary/secondary cell wall synthesizing CesAs. This suggests that they might have dual role and involved in redundant functions in cell wall biosynthesis in Leucaena. As the semi quantitative and qRT-PCR data suggests that the expression of $L l-8 C e s A$ was higher than Ll-7CesA in case of all tissues tested, it can be assumed that this isoform might be involved in active cellulose biosynthesis.

\section{ACKNOWLEDGEMENTS}

This work was supported by grants from Council of Scientific and Industrial Research-New Millennium Initiative for Technology Leadership (CSIR-NMITLI), India. We wish to thank Director, National Chemical Laboratory and Head Plant Tissue Culture Division. RKV and S. Singh thank CSIR-NMITLI for their fellowship grant and SS thanks CSIR-UGC for his fellowship grant.

\section{REFERENCES}

[1] Prasad, J.V.N.S., Korwar, G.R., Rao, K.V., Mandal, U.K., Rao, G.R., Srinivas, I., Venkateswarlu, B., Rao, S.N. and
Kulkarni H.D. (2010) Optimum stand density of Leucaena leucocephala for wood production in Andhra Pradesh, Southern India. Biomass and Bioenergy, 35, 1-9.

[2] Shelton, H.M. and Jones, R.J. (1995) Opportunities and limitations in Leucaena. Proceedings of a workshop held in Bangor, Indonesia. ACIAR Proceedings, Canberra, 1623.

[3] Saxena, I.M. and Malcolm B.J.R. (2005) Cellulose Biosynthesis: Current views and evolving concepts. Annals of Botany, 96, 9-21. doi:10.1093/aob/mci155

[4] Taylor, N.G. (2008) Cellulose biosynthesis and deposition in higher plants. New Phytologist, 178, 239-252. doi:10.1111/j.1469-8137.2008.02385.x

[5] Pear, J.R., Kawagoe, Y., Schreckengost, W.E., Delmer, D.P. and Stalker, D.M. (1996) Higher plants contain homologs of the bacterial celA genes encoding the catalytic subunit of cellulose synthase. Proceedings of the $\mathrm{Na}$ tional Academy of Sciences, 93, 12637-12642. doi:10.1073/pnas.93.22.12637

[6] Richmond, T.A. and Somerville, C.R. (2000) The cellulose synthase superfamily. Plant Physiology, 124, 495498. doi:10.1104/pp.124.2.495

[7] Appenzeller, L., Doblin, M., Barreiro, R., Wang, H.Y., Niu, X.M., Kollipara, K., Carrigan, L., Tomes, D., Chapman, M. and Dhugga, K.S. (2004) Cellulose synthesis in maize: isolation and expression analysis of the cellulose synthase (CesA) gene family. Cellulose, 11, 287-299. doi:10.1023/B:CELL.0000046417.84715.27

[8] Tanaka, K., Murata, K., Yamazaki, M., Onosato, K., Miyao, A. and Hirochika, H. (2003) Three distinct rice cellulose synthase catalytic subunit genes required for cellulose synthesis in the secondary wall. Plant Physiology, 133, 73-83. doi:10.1104/pp.103.022442

[9] Trethewey, J.A.K. and Harris, P.J. (2002) Location of $(1 \rightarrow 3),(1 \rightarrow 4)-\beta$-D-glucans in vegetative cell walls of barley (Hordeum vulgare) using immunogold labelling. New Phytologist, 154, 347-358. doi:10.1046/j.1469-8137.2002.00383.x

[10] Lu, S., Li, L., Yi, X., Joshi, C.P. and Chang, V.L. (2008) Differential Expression of Three Eucalyptus secondary cell wall-related cellulose Synthase genes in response to tension stress. Journal of Experimental Botany, 59, 681695. doi:10.1093/jxb/erm350

[11] Joshi, C.P., Bhandari, S., Ranjan, P., Kalluri, U., Liang, S., Fujino, T. and Samuga, A. (2004) Genomics of Cellulose biosynthesis in poplars. New Phytologist, 164, 53-61. doi:10.1111/j.1469-8137.2004.01155.x

[12] Kalluri, U., and Joshi, C.P. (2003) Isolation and characterization of a new, fulllength cellulose synthase cDNA from developing xylem of aspen trees. Journal of Experimental Botany, 54, 2187-2188. doi:10.1093/jxb/erg232

[13] Dhugga, K.S. (2001) Building the wall: Genes and enzyme complexes for polysaccharide synthesis. Current Opinion in Plant Biology, 4, 488-493. doi:10.1016/S1369-5266(00)00205-3

[14] Holland, D., Helentjaris, T., Dhugga, K., XoconostleCazares, B. and Delmer, D.P. (2000) A comparative 
analysis of the cellulose synthase $(\operatorname{Ces} A)$ gene family in plants. Plant Physiology, 123, 1313-1323. doi:10.1104/pp.123.4.1313

[15] Shaik, N.M., Arha, M., Nookaraju, A., Gupta, S.K., Srivastava, S., Yadav, A.K., Kulkarni, P.S., Abhilash, O.U., Vishwakarma, R.K., Singh, S., Tatkare, R., Chinnathambi, K., Rawal, S.K. and Khan B.M. (2009) Improved method of in vitro regeneration in Leucaena leucocephala-A leguminous pulpwood tree species. Physiology and Molecular Biology of Plants, 15, 311-318. doi:10.1007/s12298-009-0035-5

[16] Saitou, N. and Nei, M. (1987) The neighbor-joining method: A new method for reconstructing phylogenetic trees. Molecular Biology and Evolution, 4, 406-425.

[17] Felsenstein, J. (1985) Confidence limits on phylogenies: An approach using the bootstrap. Evolution, 39, 783-791. doi: $10.2307 / 2408678$

[18] Zuckerkandl, E. and Pauling, L. (1965) Evolutionary divergence and convergence in proteins. In: Bryson, V. and Vogel, H.J., Eds., Evolving Genes and Proteins, Academic Press, New York, 97-166.

[19] Tamura, K., Dudley, J., Nei, M. and Kumar, S. (2007) MEGA4: Molecular Evolutionary Genetics Analysis (MEGA) software version 4.0. Molecular Biology and Evolution, 24, 1596-1599. doi:10.1093/molbev/msm092

[20] Freeman, W.M., Walker, S.J. and Vrana, K.E. (1999) Quantitative RT-PCR: Pitfalls and potential. Biotechniques, 26, 112-125.

[21] Pfaffl, M.W. (2001) A new mathematical model for relative quantification Real-time RT-PCR. Nucleic Acid Research, 29, 2002-2007. doi:10.1093/nar/29.9.e45

[22] Lodhi, M.A., Ye, G.N., Weeden, N.F. and Reisch, B.I. (1994) A simple and efficient method for DNA extraction from grapevine cultivars and Vitis species. Plant Molecular Biology Reporter, 12, 6-13. doi:10.1007/BF02668658

[23] Sambrook, J., Fritsch, E.F. and Maniatis, T. (1989) Molecular cloning: A laboratory manual. 2nd Edition, Cold Spring Harbor, New York.

[24] Saxena, I.M., Brown, R.M., Fevre, M., Geremia, R.A. and Henrissat, B. (1995) Multidomain architecture of h-glycosyltransferases: Implications for mechanism of action. Journal of Bacteriology, 177, 1419-1424.

[25] Delmer, D.P. (1999) Cellulose biosynthesis: Exciting times for a difficult field of study. Annual Review of Plant Physiology and Plant Molecular Biology, 50, 245276. doi:10.1146/annurev.arplant.50.1.245

[26] Arioli, T., Peng, L., Betzner, A.S., Burn, J., Wittke, W., Herth, W., Camilleri, C., Hofte, H., Plazinski, J. and Birch, R. (1998) Molecular analysis of cellulose biosynthesis in Arabidopsis. Science, 279, 717-720.

\footnotetext{
Abbreviations

AMV-RT: Avian Myeloblastosis Virus Reverse Transcriptase.

cDNA: Complementary DNA.
}

\section{doi:10.1126/science.279.5351.717}

[27] Suzuki, M. (1989) SPXX, a frequent sequence motif in gene regulatory proteins. Journal of Molecular Biology, 207, 61-84. doi:10.1016/0022-2836(89)90441-5

[28] Hazen, S.P., Scott-Craig, J.S. and Walton, J.D. (2002) Cellulose synthase-like genes of rice. Plant Physiology, 128, 336-340. doi:10.1104/pp.010875

[29] Ranik, M. and Myburg, A.A. (2006) Six new cellulose synthase genes from Eucalyptus are associated with primary and secondary cell wall biosynthesis. Tree Physiology, 26, 545-556. doi:10.1093/treephys/26.5.545

[30] Samuga, A. and Joshi, C.P. (2002) A new cellulose synthase gene (PtrCesA2) from aspen xylem is orthologous to Arabidopsis AtCesA7 (irx3) gene associated with secondary cell wall synthesis. Gene, 296, 37-44. doi:10.1016/S0378-1119(02)00864-8

[31] Samuga, A. and Joshi, C.P. (2004) Differential expression patterns of two new primary cell wall-related cellulose synthase cDNAs, PtrCesA6 and PtrCesA7 from aspen trees. Gene, 334, 73-82. doi:10.1016/j.gene.2004.02.057

[32] Mellerowicz, E.J., Baucher, M., Sundberg, B. and Boerjan, W. (2001) Unravelling cell wall formation in the woody dicot stem. Plant Molecular Biology, 47, 239-274. doi:10.1023/A:1010699919325

[33] Taylor, N.G., Scheible, W., Cutler, S., Somerville, C.R. and Turner, S.R. (1999) The irregular xylem3 locus of Arabidopsis encodes a cellulose synthase required for secondary wall synthesis. Plant Cell, 11, 769-779.

[34] Taylor, N.G., Laurie, S. and Turner, S.R. (2000) Multiple cellulose synthase catalytic subunits are required for cellulose synthesis in Arabidopsis. Plant Cell, 12, 2529 2540 .

[35] Taylor, N.G., Howells, R.M., Hutty, A.K., Vickers, K. and Turner, S.R. (2003) Interactions among three distinct CesA proteins essential for cellulose synthesis. Proceedings of the National Academy of Sciences, 100, 14501455. doi: 10.1073 /pnas. 0337628100

[36] Joshi, C.P. (2003) Molecular biology of cellulose biosynthesis in plants. In: Pandalai, S., Ed., Recent Research Developments in Plant Molecular Biology, Research Signpost Press, Kerala, 19-38.

[37] Joshi, C.P. (2003) Xylem-specific and tension stress responsive expression of cellulose synthase genes from aspen trees. Applied Biochemistry and Biotechnology, 105, 17-26. doi:10.1385/ABAB:105:1-3:17

[38] Gardiner, J.C., Taylor, N.G. and Turner, S.R. (2003) Control of cellulose synthase complex localization in developing xylem. Plant Cell, 15, 1740-1748. doi: $10.1105 / \mathrm{tpc} .012815$

CesA: Cellulose Synthase A.

Ll-CesA: Leucaena leucocephala Cellulose Synthase A.

RACE: Rapid Amplification of cDNA Ends. qRT-PCR:Quantitative real-time PCR. 